\title{
Alcohol use and craving among Veterans with mental health disorders and mild traumatic brain injury
}

\begin{abstract}
Amy A. Herrold, PhD; ${ }^{1-2}$ Neil Jordan, PhD $;^{1-3}$ Walter M. High, PhD $;^{4-6}$ Judi Babcock-Parziale, PhD ${ }^{7}$ R. Andrew Chambers, MD $;^{8}$ Bridget Smith, PhD ${ }^{1,9-10}$ Charlesnika T. Evans, PhD $;{ }^{1,3,9}$ Xue Li, PhD ${ }^{11}$ Trudy Mallinson, PhD, OTR/L, FAOTA; ${ }^{12}$ Shonna Jenkins, MS; ${ }^{4,6}$ Theresa Louise-Bender Pape, DrPH, MA, CCCSLP/L $1,13-14$

${ }^{1}$ Center of Innovation for Complex Chronic Healthcare, Edward Hines, Jr. Department of Veterans Affairs (VA) Hospital, Hines, IL; ${ }^{2}$ Department of Psychiatry and Behavioral Sciences, Northwestern University Feinberg School of Medicine, Chicago, IL; ${ }^{3}$ Department of Preventive Medicine and Center for Healthcare Studies, Institute for Public Health and Medicine, Northwestern University Feinberg School of Medicine, Chicago, IL; ${ }^{4}$ Cardinal Hill Rehabilitation Hospital and Department of Physical Medicine and Rehabilitation, University of Kentucky College of Medicine, Lexington, KY; ${ }^{5}$ Departments of Neurosurgery and Psychology, University of Kentucky College of Medicine, Lexington, KY; ${ }^{6}$ Lexington VA Medical Center, Lexington, KY; ${ }^{7}$ Southern Arizona VA Health Care System, Tucson, AZ; ${ }^{8}$ Department of Psychiatry, Neuroscience Research Center, Indiana University School of Medicine, Indianapolis, IN; ${ }^{9}$ Spinal Cord Injury Quality Enhancement Research Initiative, Edward Hines, Jr. VA Hospital, Hines, IL; ${ }^{10}$ Child Health Research Program, Stanley Manne Children's Research Institute, Ann and Robert H. Lurie Children's Hospital of Chicago, Department of Pediatrics, Northwestern University Feinberg School of Medicine, Chicago, IL; ${ }^{11}$ Cooperative Studies Program Coordinating Center, Edward Hines, Jr. VA Hospital, Hines, IL; ${ }^{12}$ Department of Clinical Research and Leadership, The George Washington University, Washington, DC; ${ }^{13}$ Research and Development Service, Edward Hines, Jr. VA Hospital, Hines, IL; ${ }^{14}$ Department of Physical Medicine and Rehabilitation, Northwestern University Feinberg School of Medicine, Chicago, IL
\end{abstract}

\begin{abstract}
Mental health disorders (MHDs), mild traumatic brain injury (mTBI), and alcohol use disorder (AUD) are endemic among recent Veterans, resulting in a population with heterogeneous, co-occurring conditions. While alcohol craving negatively affects rehabilitation and leads to relapse, no studies have examined alcohol craving among Veterans with co-occurring MHDs and mTBI. The purpose of this preliminary cohort study is to describe alcohol craving in a convenience sample of Iraq and Afghanistan Veterans $(n=48)$, including those exposed to traumatic events and experiencing active symptoms. Veterans completed weekly telephone interviews that included the Alcohol Use Disorder Identification Test, consumption questions (AUDIT-C) (week 1) and the Penn Alcohol Craving Scale (PACS) (weeks 16). Sixty percent of the sample screened positive on the AUDIT-C for probable AUD. Using Rasch analysis, the person separation reliability of the PACS was strong (0.87) among AUDIT-C posi-
\end{abstract}

tive Veterans. Higher PACS scores were reported among AUDIT$\mathrm{C}$ positive versus AUDIT-C negative Veterans (mixed effects analysis, $p<0.001)$. PACS scores were higher among AUDIT-C positive Veterans with MHDs with and without mTBI versus AUDIT-C positive combat comparison Veterans (pairwise comparison, $p<0.001)$. Rates of hazardous alcohol use are high among Iraq and Afghanistan conflict Veterans and suggest that alcohol craving is elevated among those with MHDs with and without mTBI.

Key words: alcohol, Alcohol Use Disorder Identification Test, anxiety, craving, depression, mild traumatic brain injury, Penn Alcohol Craving Scale, posttraumatic stress disorder, psychometrics, Veteran. 


\begin{abstract}
Abbreviations: $\mathrm{ANOVA}=$ analysis of variance; $\mathrm{AUD}=$ alcohol use disorder; AUDIT-C = Alcohol Use Disorder Identification Test, consumption questions; BAI = Beck Anxiety Inventory; BDI-II = Beck Depression Inventory-II; CAPS = Clinician-Administered Posttraumatic Stress Disorder Scale; $\mathrm{CC}=$ combat comparison; $\mathrm{CI}=$ confidence interval; $\mathrm{HSD}=$ honest significant difference; IRB $=$ Institutional Review Board; IVR $=$ interactive voice response; $\mathrm{MHD}=$ mental health disorder; $\mathrm{MNSQ}=$ mean square; $\mathrm{mTBI}=$ mild traumatic brain injury; PACS = Penn Alcohol Craving Scale; PNS = Polytrauma Network Site; PSR = person separation reliability; PTSD = posttraumatic stress disorder; SI = separation index; $\mathrm{VA}=$ Department of Veterans Affairs.

Address all correspondence to Amy A. Herrold, PhD; 5000 S Fifth Ave, MC 151H, Edward Hines, Jr. VA Hospital, Hines, IL 60141; 708-202-5867; fax: 708-202-7487.

Email: Amv.Herrold@va.gov (within United States), Amy.Herrold@northwestern.edu (outside United States) http://dx.doi.org/10.1682/JRRD.2013.07.0170
\end{abstract}

\section{INTRODUCTION}

Hazardous alcohol use, indicative of alcohol use disorder (AUD), is endemic among U.S. Veterans deployed to Iraq and Afghanistan [1-3]. AUD rates are even higher for Veterans returning from deployment with co-occurring mental health disorders (MHDs) and mild traumatic brain injury (mTBI) [4-6]. In fact, occurrence of MHDs or mTBI is a known risk factor for AUD [7-8]. Increased risk for AUD is clinically important because alcohol misuse detracts from therapeutic efforts targeting MHDs and mTBI-related impairments [9-10].

Intuitively, it is plausible that the co-occurrence of MHDs and mTBI contributes to increased vulnerability to AUD due to craving. The interrelationship of these cooccurring conditions with alcohol craving has received little research attention. Alcohol craving is a well-established risk factor for AUD relapse [11-12]. There is some evidence suggesting that alcohol craving is more severe among individuals with MHDs (e.g., depression, anxiety, and posttraumatic stress disorder [PTSD]) [13-15], but no studies to date have examined alcohol craving among Veterans with co-occurring MHDs, mTBI, and AUD. Because the co-occurrence of MHDs, mTBI, and AUD is common among U.S. Veterans deployed to Iraq and Afghanistan and evidence suggests that alcohol craving may be exacerbated by these conditions, we sought to describe alcohol craving among a group of Veterans exposed to traumatic events during deployment who reported active MHD or mTBI symptoms at the time of enrollment into a larger parent study. The purpose of this article is to report our descriptive findings, which will enable future research that addresses the need to elucidate the relationships among MHDs, mTBI, AUD, and alcohol craving.

\section{Prevalence of and Risk for Co-occurring Mild Trau- matic Brain Injury, Mental Health Disorders, and Alcohol Use Disorder}

Mild TBI is often referred to as the signature injury of the conflicts in Iraq and Afghanistan, in part, because mTBI prevalence rates, ranging from 16 to 20 percent, are higher than rates reported for other conditions among these same Veterans [16-17]. Among Veterans with mTBI, depression and anxiety disorders such as PTSD are the most commonly co-occurring MHDs. Prevalence rates for PTSD and depression, for example, have been reported to be as high as 44 and 23 percent, respectively [18-19].

Evidence indicates that people with mTBI are at increased risk for developing postinjury AUD [7] and that Veterans with PTSD or depression have 3.0 to 4.5 times greater risk of being diagnosed with AUD [2]. Furthermore, Veterans with mTBI and AUD have a higher incidence of mood disorders $(60 \%)$ relative to Veterans with TBI but no AUD (37\%) [10].

\section{Relationship Between Alcohol Use Disorder and Alcohol Craving}

Alcohol craving, the urge to drink, is associated with an increased risk of relapse among people with AUD [11-12,20-21]. More severe [13] and more frequent [14] alcohol craving is reported by people with PTSD and cooccurring AUD relative to people with AUD alone. Increased frequency and severity of alcohol craving are also linked with the incidence of depression [15,22] and more severe depressive symptoms [23].

Collectively, the evidence suggests that people with co-occurring mTBI, MHDs, and AUD conditions may be more susceptible to AUD relapse. Little is known, however, about the frequency and severity of alcohol craving among people with these co-occurring conditions. Since alcohol misuse detracts from rehabilitation efforts, a better understanding of the frequency and severity of alcohol craving among Veterans with co-occurring mTBI, MHDs, and AUD will inform clinical rehabilitation efforts. 
To address the need for a better understanding of alcohol craving among Veterans deployed to Iraq and/or Afghanistan who present with co-occurring mTBI, MHDs, and AUD, we examined self-reported alcohol craving in a sample of Veterans with active MHD symptoms with and without mTBI symptoms. For comparison purposes, we also examined self-reported alcohol craving among a sample of healthy, asymptomatic Veterans also deployed to Iraq and/or Afghanistan (i.e., combat comparison group). Because alcohol craving has not been assessed in this population, we also sought to describe the construct validity and measurement precision of a self-report alcohol craving instrument, the Penn Alcohol Craving Scale (PACS). We hypothesized that (1) the PACS items would form a single scale of alcohol craving that could distinguish among Veterans with different levels of craving and (2) alcohol craving would be more frequent and more severe among Veterans with MHDs, both with and without $\mathrm{mTBI}$, relative to a combat comparison group with AUD alone.

\section{METHODS}

\section{Study Design}

This was a pilot study of U.S. Veterans deployed during the Iraq and Afghanistan conflicts who were recruited from three Department of Veterans Affairs (VA) Polytrauma Network Sites (PNSs) between August 2011 and December 2012 to participate in a cross-sectional study examining the diagnostic accuracy for the VA's Traumatic Brain Injury Clinical Reminder and Comprehensive Traumatic Brain Injury Evaluation processes (VA Health Services Research and Development ServiceDirected Research grant SDR-08-377). The parent research study was approved by the VA Central Institutional Review Board (IRB), and the informed consent provided participants with the option to be contacted about a future research study. The present, prospective descriptive pilot study enrolled 52 participants from the parent study and was approved by the Human Subjects IRB at the Edward Hines, Jr. VA Hospital. A separate consent form was used for participation in this additional research study. A sample size of 50 was proposed for the current study based on both budgetary constraints and study goals. To enable reasonable estimates of item locations in Rasch analysis of polytomous items, 10 observations per rating scale step have been shown to be acceptable for preliminary investigations [24], although these results should be interpreted cautiously [25].

\section{Testing Battery}

Participation in the parent study involved extensive neuropsychological tests, mental health symptom inventories, self-report questionnaires, and quality of life assessments, which were used to classify each Veteran into a diagnostic group (i.e., mTBI only, MHD only, mTBI+MHD, exposed asymptomatic controls). Classification for the parent study was based on an algorithm developed by clinical experts on the research team (see Appendix, available online only).

The assessment battery for the parent, cross-sectional study included tests to identify Veterans with MHDs and to differentially diagnose the type of MHD. For the purposes of this study, a MHD includes depression, anxiety, and/or PTSD symptoms that meet the following study criteria. A Beck Depression Inventory-II (BDI-II) [26-27] score of 17 or higher, a Beck Anxiety Inventory (BAI) [28-29] score of 8 or higher, and/or meeting the lenient criteria [30] on the Clinician-Administered Posttraumatic Stress Disorder Scale (CAPS) classified Veterans in the MHD group. Criteria to be classified as having mTBI included reports indicating currently active symptoms (e.g., headache, sensitivity to light, and lack of concentration) where symptoms were corroborated by performance on neuropsychological tests. Three classification groups were identified: (1) MHD and mTBI (MHD+mTBI), (2) MHD but no mTBI (MHD), and (3) neither MHD nor mTBI (combat comparison [CC] group). Clinical neuropsychologists verified each MHD and mTBI classification according to the aforementioned diagnostic algorithm. Demographic information and time since injury (where relevant) were also obtained for each research participant.

\section{Study Sample}

The cohort for the parent, cross-sectional study included 438 Veterans, including those seeking care at three VA PNSs and Veterans from the surrounding communities. To create a cohort for the present study (Figure 1), we contacted Veterans from two of the three original study sites as dictated by pilot study budget constraints. The two sites chosen, one large, urban VA hospital and one midsized, rural VA medical center, had the greatest number of Veterans in the three classification groups of interest described in the previous paragraph. In all, 246 of the 307 


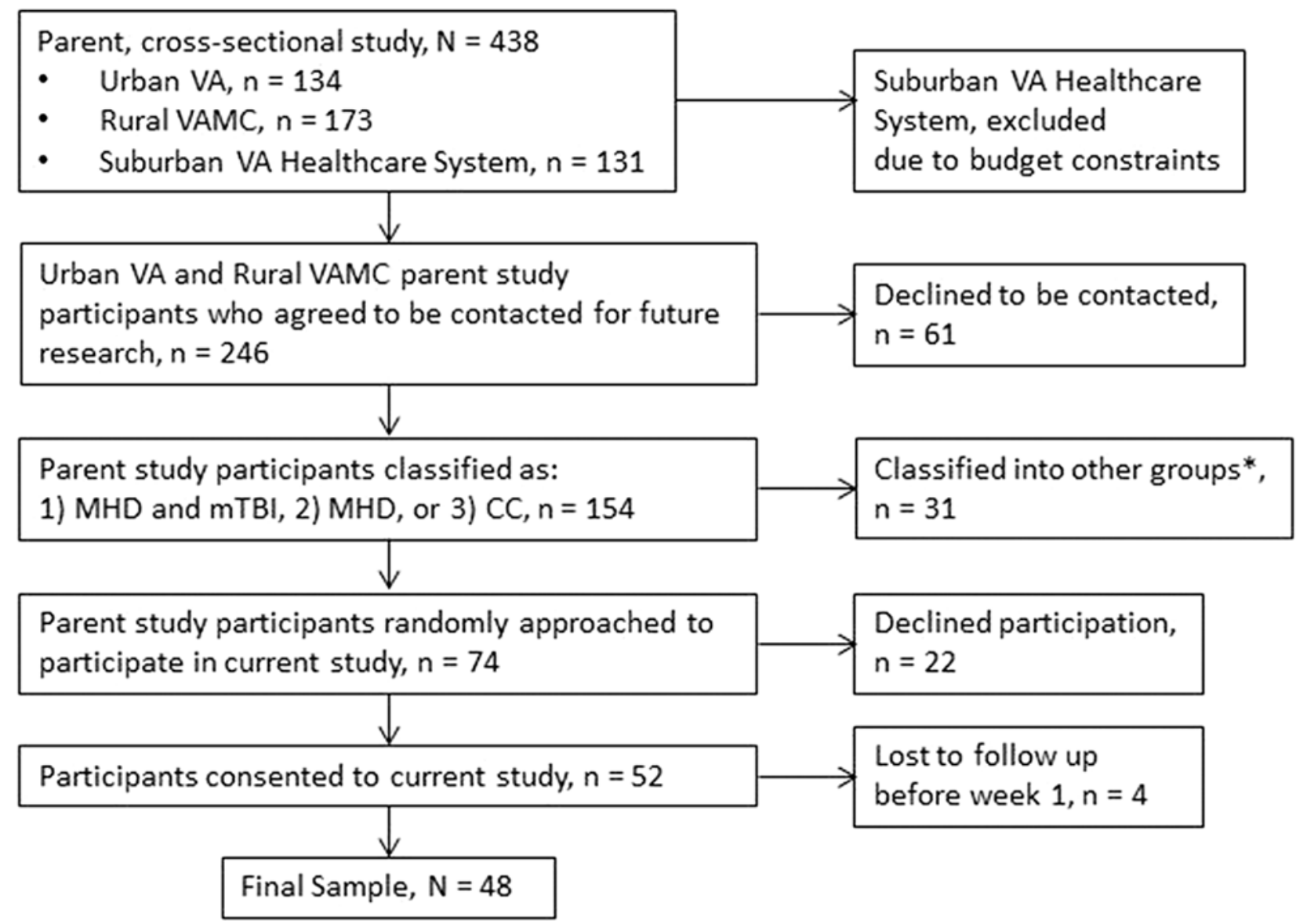

Figure 1.

Study sample flow diagram. *Other classification groups from parent study that were not of relevance to current study included symptom resolution, questionable validity, and other unrelated conditions. $C C=$ combat comparison, $\mathrm{MHD}=$ mental health disorder, $\mathrm{mTBI}=$ mild traumatic brain injury, VA = Department of Veterans Affairs (hospital), VAMC = VA medical center.

Veterans from these two sites who participated in the parent study consented to being contacted for participation in additional research. Of these 246 Veterans, 154 were classified into the three classification groups. All 154 Veterans were sent a letter inviting them to participate in the study. A convenience sample of 74 of these Veterans was contacted regarding study participation, beginning with Veterans at the urban VA hospital. In line with the study sample size goal described earlier, recruitment calls were concluded when 52 Veterans had consented to participate. To minimize the possibility of perceived coercion, invited Veterans were not asked to provide a reason for declining participation. After consent, four Veterans could not be reached to complete the first week of telephone questionnaires and were dropped from the study, resulting in a final sample of 48 Veterans.

\section{Data Collection Procedures for Additional Research Participation}

All participants completed self-reported alcohol use and alcohol craving questionnaires via telephone interview weekly for 6 wk. Telephone interviews during weeks 1 and 6 lasted approximately $30 \mathrm{~min}$; these interviews included verbal administration of the Alcohol Use Disorders Identification Test, consumption questions (AUDIT-C) and the PACS and scheduling of the next weekly call. Interviews during weeks $2-5$ each lasted approximately $5 \mathrm{~min}$ and included verbal administration of the PACS only. Administration of the PACS over the telephone to assess self-reported alcohol craving among Veterans including those with PTSD has been previously reported by Simpson et al. [31]. The Simpson et al. study administered the PACS weekly over the telephone with 
interactive voice response (IVR) technology. We adapted this existing protocol by administration of the PACS over the telephone by a research assistant. We also adapted the compensation schedule from the Simpson et al. study, which resulted in good compliance with research procedures [31], and compensated Veterans for the number of weekly telephone calls they completed. Compensation increased from $\$ 10$ for completion of the week 1 interview only to $\$ 74$ for the completion of all $6 \mathrm{wk}$ and was prorated for participants completing weeks $2-5$.

\section{Instrumentation}

\section{Penn Alcohol Craving Scale}

The PACS is a five-item, self-report assessment of alcohol craving [32] for which lower total scores indicate less frequent and less severe craving and higher scores indicate more frequent and more severe craving. Prior studies indicate strong internal consistency for the PACS (Cronbach $\alpha=0.92$ ) [32]. The PACS has also been reported to show concurrent and discriminant validity in a population of individuals with AUD only [32]. Finally, PACS scores demonstrate predictive validity among individuals with AUD only via a significant relationship with relapse [32-33]. PACS telephone administration to assess self-reported alcohol craving among Veterans, including those with PTSD, has been previously reported by Simpson et al. [31].

\section{Alcohol Use Disorder Identification Test, Consumption Questions}

The AUDIT-C is a three-item, self-report measure of alcohol use [34-36]. A score of 4 or more for men and 3 or more for women on this assessment indicates hazardous drinking and probable AUD. Using these cutoffs, the AUDIT-C has 86 percent sensitivity and 72 percent specificity for accurately identifying people with AUD [34].

\section{Study Sample Groups}

Because this investigation focused on the relationship between alcohol craving and alcohol use in Veterans with and without active symptoms, we combined participants with MHD and MHD+mTBI. We believed this to be reasonable because no differences were found between these groups based on demographic factors including age, sex, race, ethnicity, education, and marital status. Furthermore, there were no differences found between groups on baseline mental health symptom assessments (BDI-II, BAI, and CAPS). However, there was a significant difference in employment status between the two groups (Fisher exact test, $p=0.045$ ), with a higher proportion of individuals among the MHD group working than in the MHD+mTBI group.

Veterans were further classified according to selfreported alcohol use on the AUDIT-C. The following four groups detailed in Table 1 were compared for this descriptive study: (1) MHD \pm mTBI, AUDIT-C negative (-); (2) MHD \pm mTBI, AUDIT-C positive (+); (3) CC, AUDIT-C(-); and (4) CC, AUDIT-C(+).

\section{Data Analyses}

Since the PACS has not been used with Veterans with mTBI with or without co-occurring conditions, the psychometric properties of the PACS ratings scale and test items were examined prior to using the scores in the correlational analyses. Using Winsteps ${ }^{\circledR}$ software version 3.74.0 (Winsteps, Inc; Chicago, Illinois) [37], we used a partial credit Rasch model [38] to examine psychometric properties of the PACS measure in the AUDIT-C $(+)$ population of Veterans, including those with MHD with and without mTBI. Psychometric properties of the PACS were examined using the first week of participant data because responses were not expected to change over time [31]. We examined the reliability, precision, item hierarchy, and structure of the PACS rating scale for each test item to determine whether they proceeded in order

Table 1.

Description of study groups.

\begin{tabular}{lrl}
\hline \multicolumn{1}{c}{ Group Name } & $\boldsymbol{n}$ & \multicolumn{1}{c}{ Group Description } \\
\hline MHD \pm mTBI, AUDIT-C $(-)$ & 6 & Veterans with MHD with and without mTBI who screened negative on AUDIT-C. \\
MHD \pm mTBI, AUDIT-C $(+)$ & 15 & Veterans with MHD with and without mTBI who screened positive on AUDIT-C. \\
$\mathrm{CC}$, AUDIT-C $(-)$ & 13 & CC Veterans who screened negative on AUDIT-C. \\
$\mathrm{CC}$, AUDIT-C $(+)$ & 14 & CC Veterans who screened positive on AUDIT-C. \\
\hline
\end{tabular}

AUDIT-C $=$ Alcohol Use Disorder Identification Test, consumption questions; $\mathrm{CC}=$ combat comparison; MHD $=$ mental health disorder; $\mathrm{mTBI}=$ mild traumatic brain injury. 
(monotonic). We examined overall assessment psychometrics including person separation reliability (PSR) and the person separation index (SI). PSR is analogous to Cronbach alpha, and values can be interpreted in the same manner (i.e., PSR values greater than 0.8 should be considered satisfactory) [39]. The SI is an indication of measurement precision, and this index indicates the number of performance or ability levels the scale is able to identify [40]. We also examined the fit of each test item and each person to the underlying assumptions of the measurement model. Infit mean square (MNSQ) standardized residuals indicate the extent to which items capture similar constructs to that of other items in the scale. Values between 0.7 and 1.3 were considered acceptable [41-42].

\section{Group Comparisons}

Each of the four study groups was compared on baseline demographic variables, mental health variables, and alcohol use (AUDIT-C scores). For continuous baseline variables, one-way analysis of variance (ANOVA) was used to determine between-group differences. When overall group comparisons were significant $(\alpha=0.05)$, pairwise comparison tests (Tukey test for multiple comparisons) were also conducted. For categorical baseline variables, a Fisher exact test (due to insufficient cell size) was used to determine between-group differences.

To analyze PACS scores over $6 \mathrm{wk}$, a mixed-effects regression model with a random intercept and a linear time trend [43] was used; this model treats time as a continuous variable and explicitly models individual change over time. A restricted maximum likelihood estimation method accounting for small group size was employed. This approach uses all available observed data points and assumes that missing data points occurred in a random manner. Covariates tested in the mixed-effects model included time, group (three dummies that contrast each of the other groups to MHD \pm mTBI, AUDIT-C $[+]$ ), time $\times$ group interaction, and baseline demographic and/or mental health covariates that were significantly different $(p<$ 0.05 ) across the four groups. Model selection was based on the Bayesian Information Criteria. Statistical analyses were completed using SAS (SAS Institute Inc; Cary, North Carolina) and SPSS (IBM Corporation; Armonk, New York). All statistical tests were two-sided at $\alpha=$ 0.05 level. The criterion for statistical significance was $p<0.05$.

\section{RESULTS}

\section{Penn Alcohol Craving Scale Rating Scale and Reli- ability}

The PACS rating scale steps for each of the test items proceeded monotonically, indicating that lower-rating categories were, as theorized, more probable for people with less severe craving, and the higher-rating category was, as theorized, more probable for people with more severe craving. Since inclusion of AUDIT-C(-) Veterans would not add information about functioning of a test for patients with AUD, we examined measurement reliability and precision of the PACS only for the AUDIT-C $(+)$ Veterans $(n=29 / 48$ Veterans). Test measurement reliability and precision with this AUDIT-C $(+)$ population was strong, as indicated by PSR of 0.87 and item SI of 2.61 . These indices mean that the PACS responses had a high level of precision in distinguishing different degrees of craving among AUDIT-C $(+)$ Veterans.

The hierarchical order of the item calibrations reflects the challenge of the items from easiest to hardest to endorse, and an item map was generated (Figure 2) to illustrate the ordering of the items relative to the craving levels reported by the Veterans. This map illustrates, for example, that the study Veterans found it easiest to report difficulty resisting taking a drink (item 4), time spent thinking about craving (item 3), and frequent cravings (item 1). In summary, the rating scale functioned as theorized for this sample of Veterans with co-occurring MHDs and mTBI.

Examination of construct validity of each of the items, via an examination of infit MNSQ statistics, indicates that four of the five test items (frequency, duration, intensity, and overall craving) fit the measurement model (MNSQ = $0.87,0.96,0.90$, and 0.70 , respectively). One item, difficulty resisting taking a drink, had an infit MNSQ value of 1.42 , suggesting that responses to this item were somewhat more erratic than expected by the model. Removing this item did not improve the overall assessment psychometrics significantly ( $\mathrm{SI}=2.75)$, so this item was retained in the analyses. A greater-than-expected number of Veterans misfit (14\%) the model, which may be a result of the heterogeneous sample. In summary, the rating scale and items of the PACS functioned as theorized for this sample of Veterans with co-occurring MHDs and mTBI. 


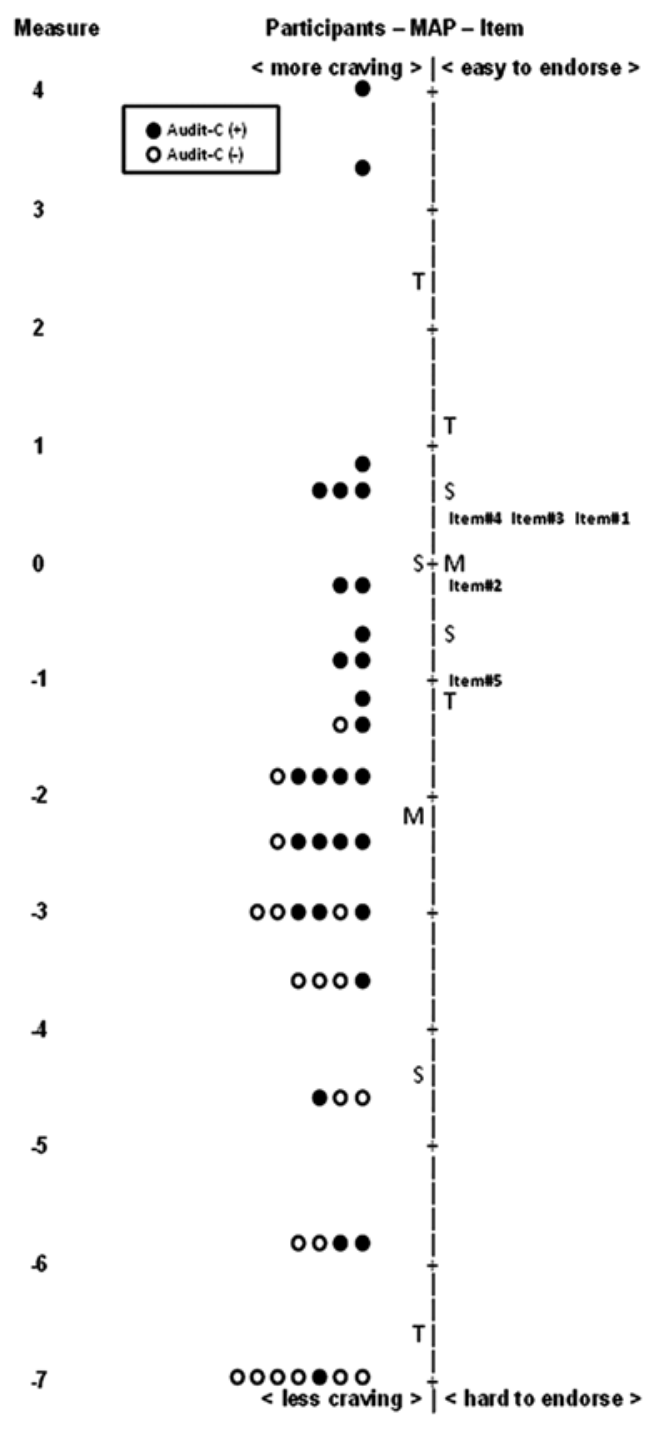

Figure 2.

Participant-item map of Penn Alcohol Craving Scale (PACS). Participants are on left of dashed line. Filled circles indicate Alcohol Use Disorder Identification Test, consumption questions (AUDIT-C) positive (+) participants and empty circles indicate AUDIT-C negative (-) participants. Items are located on right of dashed line, with items that are harder to endorse located at bottom and items that are easier to endorse located at top of map. Item 1: How often have you thought about drinking? Item 2: How strong was your craving? Item 3: How much time have you spent thinking about drinking? Item 4: How difficult would it have been to resist taking a drink? Item 5: Overall average alcohol craving. Higher scores indicate more severe craving. $\mathrm{M}=$ mean, $S=1$ standard deviation from mean, $T=2$ standard deviations from mean.

\section{Description of Study Sample}

Table 2 summarizes the demographic characteristics of the study sample and each of the four diagnostic groups. The Veteran study sample reflects the population of deployed military personnel, which is predominantly young (31 $\pm 9 \mathrm{yr}$ of age), white $(77.1 \%)$, non-Hispanic $(75.0 \%)$, and male $(92.0 \%)$. The MHD \pm mTBI, AUDIT$\mathrm{C}(-)$ group included one person with anxiety symptoms alone; one person with depression and anxiety symptoms; one person with anxiety symptoms, PTSD, and mTBI; one person with mTBI, depression symptoms, and PTSD; and two people with depression symptoms, anxiety symptoms, PTSD, and mTBI. The MHD \pm mTBI, AUDIT-C $(+)$ group included three people with anxiety symptoms alone; two people with depression and anxiety symptoms; one person with depression symptoms and PTSD; one person with anxiety symptoms and PTSD; four people with depression symptoms, anxiety symptoms, and PTSD; one person with depression symptoms and mTBI; one person with depression symptoms, PTSD, and mTBI; and two people with depression symptoms, anxiety symptoms, PTSD, and mTBI (data not shown).

The participants in the MHD $\pm \mathrm{mTBI}$ groups who had experienced mTBI $(n=8 / 21)$ were enrolled in this study an average of $42 \pm 24$ mo since time of last injury (Table 3). Four of these participants did not have probable AUD (AUDIT-C[-]), and four participants were positive for probable AUD (AUDIT-C[+]). The Veterans with AUD (AUDIT-C $[+]$ ) were enrolled into the study at a similar time postinjury $(28 \pm 27 \mathrm{mo})$ relative to the Veterans who did not have AUD (AUDIT-C[-]), $55 \pm 11 \mathrm{mo}$, two-sample $t$-test, $p=0.17$.

Mental health symptoms and scores according to the BDI-II, BAI, and CAPS were used to classify Veterans. Therefore, these scores were not treated as outcomes. However, they are presented for descriptive purposes in Table 3. As expected, the MHD \pm mTBI Veterans were, at time of enrollment into the larger study, experiencing symptoms of depression, anxiety, and/or PTSD.

The four groups were not significantly different according to age (one-way ANOVA, $F_{[3,33]}=1.004, p=$ $0.40)$, sex (Fisher exact test, $p=0.59)$, race $(p=0.52)$, ethnicity $(p=0.46)$, premilitary education $(p=0.22)$, or marital status $(p=0.36)$. There was a significant difference, however, in employment status between groups (Fisher exact test, $p=0.03$ ). The CC, AUDIT-C(-) group 
JRRD, Volume 51, Number 9, 2014

Table 2.

Demographic characteristics of study sample.

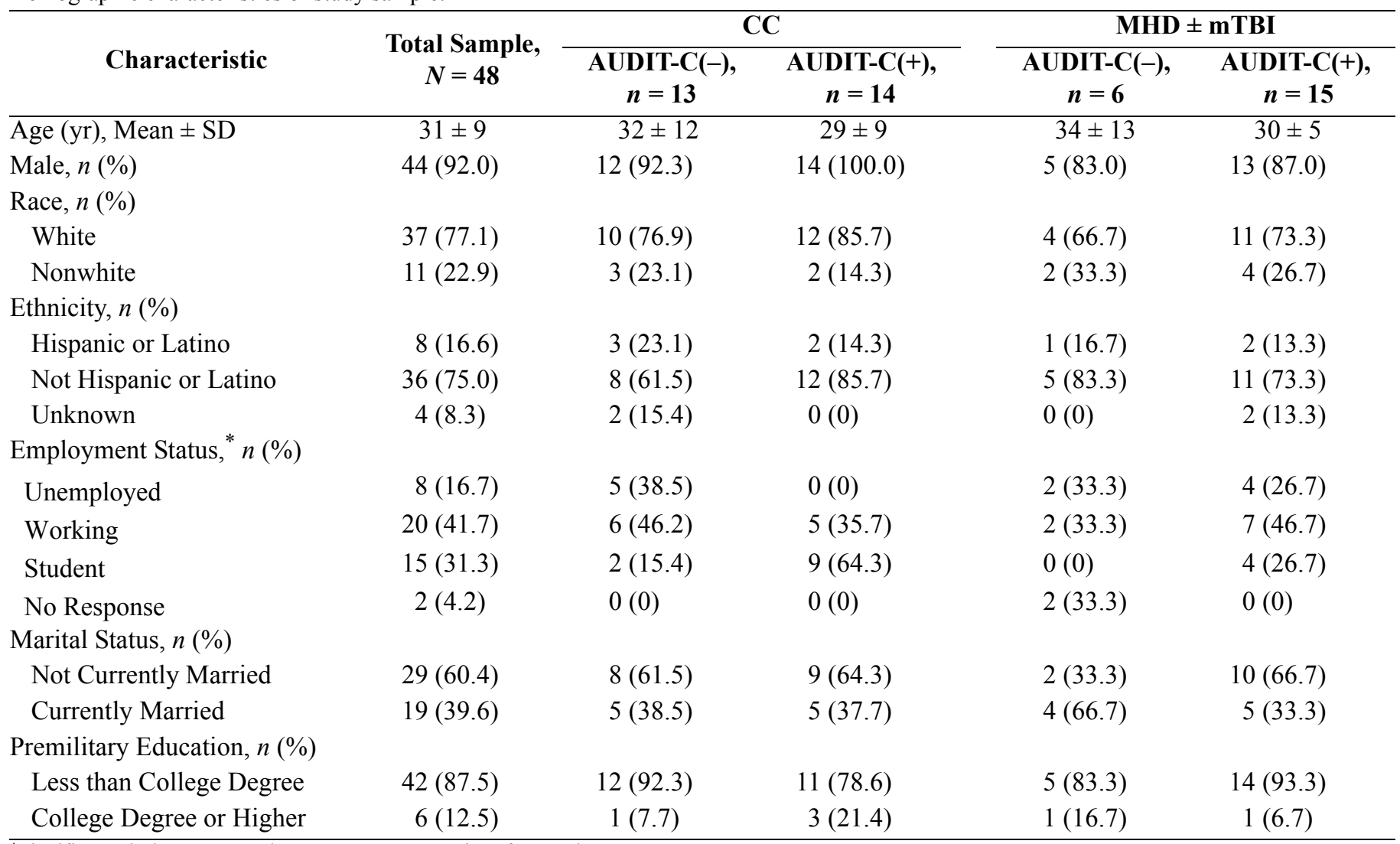

${ }^{*}$ Significant Fischer exact test between group comparison for Employment Status.

AUDIT-C = Alcohol Use Disorder Identification Test, consumption questions; $\mathrm{CC}=$ combat comparison; $\mathrm{MHD}=$ mental health disorder; $\mathrm{mTBI}=$ mild traumatic brain injury; $\mathrm{SD}=$ standard deviation.

Table 3.

Study sample injury, mental health, and alcohol use characteristics.

\begin{tabular}{|c|c|c|c|c|c|}
\hline \multirow[b]{2}{*}{ Characteristic } & \multirow{2}{*}{$\begin{array}{l}\text { Total Sample, } \\
\qquad N=48\end{array}$} & \multicolumn{2}{|c|}{$\mathbf{C C}$} & \multicolumn{2}{|c|}{ MHD \pm mTBI } \\
\hline & & $\begin{array}{l}\text { AUDIT-C(-), } \\
\quad n=13\end{array}$ & $\begin{array}{l}\text { AUDIT-C(+), } \\
\quad n=14\end{array}$ & $\begin{array}{l}\text { AUDIT-C(-) } \\
\quad n=6\end{array}$ & $\begin{array}{c}\text { AUDIT-C(+), } \\
n=15\end{array}$ \\
\hline $\begin{array}{l}\text { Time Since Injury (mo), } \\
\text { Mean } \pm \text { SD }\end{array}$ & $\begin{array}{c}42 \pm 24 \\
\text { (for } 8 \text { with } \mathrm{mTBI} \text { ) }\end{array}$ & NA & NA & $\begin{array}{c}55 \pm 11 \\
\text { (for } 4 \text { with } \mathrm{mTBI})\end{array}$ & $\begin{array}{c}28 \pm 27 \\
\text { (for } 4 \text { with } \mathrm{mTBI} \text { ) }\end{array}$ \\
\hline BDI-II, Mean \pm SD & $10.4 \pm 9.6$ & $4.9 \pm 4.6$ & $4.5 \pm 4.5$ & $19.2 \pm 11.8^{* *}, \# \#$ & $17.1 \pm 8.7^{* *}$ \\
\hline $\mathrm{BAI}$, Mean $\pm \mathrm{SD}$ & $7.5 \pm 8.9$ & $1.5 \pm 2.1$ & $2.2 \pm 2.2$ & $16.3 \pm 10.4^{* * *}, \# \# \#$ & $14.0 \pm 9.0^{* * *}, \# \# \#$ \\
\hline $\mathrm{CAPS},{ }^{\S} n(\%)$ & $13(27)$ & $0(0)$ & $0(0)$ & $4(67)$ & $9(60 s)$ \\
\hline $\begin{array}{l}\text { AUDIT-C Score, } \\
\text { Week } 1, \text { Mean } \pm \text { SD }\end{array}$ & $4.6 \pm 3.0$ & $1.9 \pm 0.9$ & $6.3 \pm 2.1^{* * *}$ & $1.3 \pm 0.5^{\# \# \#}$ & $6.7 \pm 2.4^{* * *, \dagger \dagger \dagger}$ \\
\hline $\begin{array}{l}{ }^{*} \text { Significant difference from CC } \\
{ }^{\#} \text { Significant difference from CC } \\
{ }^{\dagger} \text { Significant difference betweer } \\
\text { comparisons. } \\
{ }^{\S} \text { Significant Fisher exact test b } \\
\text { AUDIT-C = Alcohol Use Diso } \\
\text { Clinician-Administered Posttra } \\
\text { not applicable; SD = standard c }\end{array}$ & $\begin{array}{l}\text { AUDIT-C(-) group; }{ }^{* *} p \\
\text { AUDIT-C (+) group; }{ }^{* \#} \\
\text { MHD } \pm \text { mTBI, AUDIT- } \\
\text { ween group comparison } \\
\text { der Identification Test, c } \\
\text { matic Stress Disorder Sc } \\
\text { viation. }\end{array}$ & $\begin{array}{l}<0.01,{ }^{* * *} p<0.001 \\
<0.01,{ }^{* \#} p<0.001 \\
(-) \text { and MHD } \pm \mathrm{mT} \\
\text { or CAPS. } \\
\text { insumption question } \\
\text { le; } \text { CC }=\text { combat co }\end{array}$ & $\begin{array}{l}\text { AUDIT-C }(+) \text { grour } \\
\text { BAI = Beck Anxiety } \\
\text { arison; MHD = men }\end{array}$ & $\begin{array}{l}\text { t } p<0.001 \text {, according to po } \\
\text { entory; BDI-II = Beck Dep } \\
\text { ealth disorder; } \mathrm{mTBI}=\text { mild }\end{array}$ & $\begin{array}{l}\text { hoc Tukey test for multiple } \\
\text { sion Inventory-II; CAPS = } \\
\text { aumatic brain injury; NA = }\end{array}$ \\
\hline
\end{tabular}


was more likely to be unemployed (38.5\%). Most CC, AUDIT-C $(+)$ Veterans $(64.3 \%)$ were students, while students comprised a minority in the other groups.

\section{Alcohol Use}

The majority of the study sample $(29 / 48 ; 60 \%)$ screened positive for probable AUD. Mean AUDIT-C scores from week 1 of study participation are reported in Table 3. There was a significant difference between groups $\left(F_{[3,47]}=26.63, p<0.001\right)$, where AUDIT-C scores were significantly higher in the $\mathrm{CC}$, AUDIT-C $(+)$ group than in the $\mathrm{CC}$, AUDIT-C(-) and $\mathrm{MHD} \pm \mathrm{mTBI}$, AUDIT-C(-) groups (Tukey honest significant difference [HSD], $p<0.001)$. AUDIT-C scores for the MHD \pm mTBI, AUDIT-C $(+)$ group were also higher than for the $\mathrm{CC}$, AUDIT-C(-) and MHD \pm mTBI, AUDIT-C(-) groups (Tukey HSD, $p<0.001$ ). Importantly, there was no difference in AUDIT-C scores between the CC, AUDIT-C $(+)$ and $\mathrm{MHD} \pm \mathrm{mTBI}$, AUDIT-C $(+)$ groups $(p>0.05)$.

\section{Alcohol Craving}

Repeated measure analyses of PACS measures of alcohol craving severity (Figure 3) indicate that there was an overall significant effect of group $(p<0.001)$. Pairwise comparisons demonstrated that there was a significant increase in PACS alcohol craving severity measures for the MHD \pm mTBI, AUDIT-C $(+)$ group compared with the MHD \pm mTBI, AUDIT-C $(-)$ group (95\% simultaneous confidence interval [CI] of the difference: $4.95-13.54, p<0.001)$. There was also a significant increase in PACS craving severity measures reported by the $\mathrm{MHD} \pm \mathrm{mTBI}$, AUDIT-C $(+)$ group compared with the $\mathrm{CC}$, AUDIT-C $(+)$ group $(95 \%$ simultaneous $\mathrm{CI}$ of the difference: $3.43-10.07, p<0.001)$.

There was no significant effect of time $(p=0.50)$ or group $\times$ time interaction $(p=0.85)$. When adjusting for baseline demographic covariates, analyses indicate that none of the potential demographic covariates of alcohol craving, including age, race, ethnicity, employment status, premilitary education, and marital status, were significant when time and group were in the estimation model.

\section{DISCUSSION}

The study represents the first examination of the frequency and severity of alcohol craving among a group of Veterans with MHDs and co-occurring mTBI. The

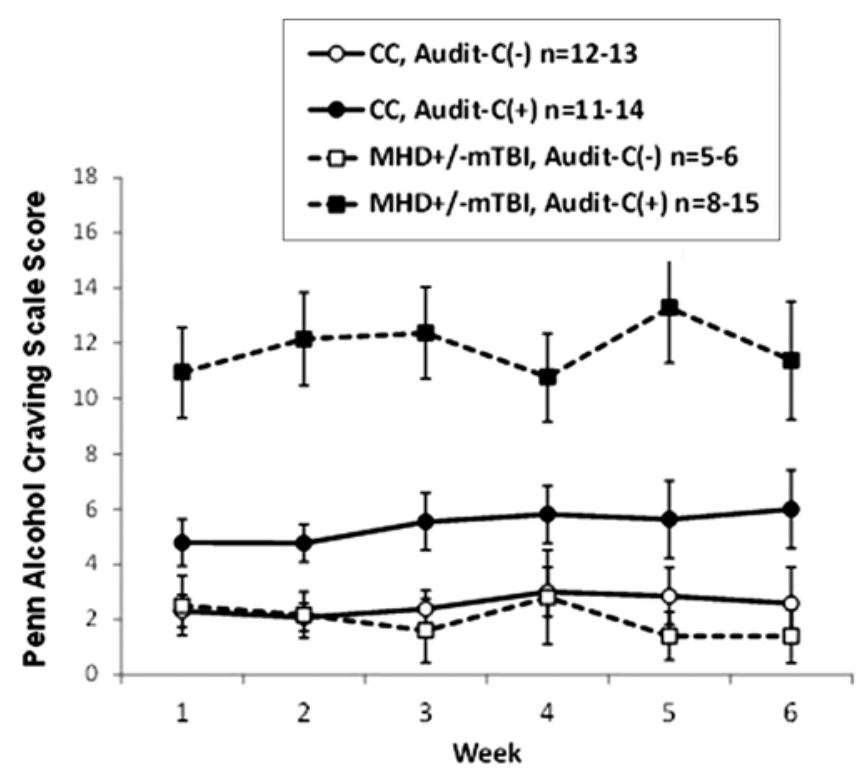

Figure 3.

Alcohol craving in Iraq and Afghanistan conflict Veterans with and without history of mental health disorders (MHDs) and mild traumatic brain injury $(\mathrm{mTBI})$. There was significant effect of group $(p<0.001)$ and no effect of time $(p=0.50)$ on selfreported alcohol craving assessed with Penn Alcohol Craving Scale (PACS). PACS scores were significantly higher for MHD \pm mTBI, Alcohol Use Disorder Identification Test, consumption questions (AUDIT-C) positive $(+)$ Veterans (black filled squares, $n=8-15)$ than for MHD $\pm \mathrm{mTBI}$, AUDIT-C negative $(-)$ Veterans (white empty squares, $n=5-6), p<0.001$. PACS scores were significantly higher for $\mathrm{MHD} \pm \mathrm{mTBI}, \mathrm{AUDIT}-\mathrm{C}(+)$ Veterans (black filled squares, $n=8-15$ ) than for CC, AUDIT-C(+) Veterans (black filled circles, $n=11-14$ ), $p<0.001$.

reported findings are descriptive and make an important contribution to the field of addiction and rehabilitation because the study sample is representative of the heterogeneity and complexity of co-occurring neurological and mental health conditions that are so common among Veterans of the Iraq and Afghanistan conflicts. As expected, Veterans with probable AUD reported more frequent and more severe alcohol craving than Veterans who screened negative for probable AUD. Furthermore, among Veterans with probable AUD, those with MHDs with and without mTBI reported more frequent and more severe alcohol craving relative to asymptomatic CC Veterans. These findings indicate that, as expected, increased alcohol use and history of MHDs with and without mTBI in 
this sample are associated with more severe and more frequent alcohol cravings.

The rates of hazardous alcohol use and probable AUD in our sample $(60 \%)$ were higher than reported findings from a larger sample $(N=585)$ of National Guard Veterans returned from Iraq and Afghanistan $(36 \%)$ [1] or among Iraq and Afghanistan Veterans $(13 \%-40 \%)[2-3]$. This could be due to disparity in sample sizes between the current study $(N=48)$, or it could also be because some Veterans in the parent study were recruited through VA PNSs and, therefore, more likely to be seeking treatment.

Our findings suggest that it may be useful to assess alcohol craving among Veterans returning from Iraq and Afghanistan by using an instrument such as the PACS. Thus, future work will be needed to validate these findings in a larger population to demonstrate utility of the PACS. These findings indicate that the PACS has the potential to be used as both an assessment tool and as an outcome measure. Because the PACS is brief (5 items), can be repeated over time, and can be administered in multiple settings (e.g., over the telephone or in person by the patient), the PACS is well suited for clinical use. The PACS can be administered by anyone in the clinic because it does not require advanced training to administer. Clinicians could easily implement the PACS during an initial assessment and over time to assess treatment progression.

Our psychometric examination of the PACS indicates a more than satisfactory level of reliability and precision for the Veteran sample. The usefulness of the PACS is also illustrated by our results that the MHD \pm mTBI Veterans who were AUDIT-C(+) had significantly higher PACS scores than their AUDIT-C(-) counterparts and by the strong reliability of the PACS $(\mathrm{PSR}=0.92)$ in our AUDIT-C $(+)$ Veterans. Furthermore, our results show that as a whole, the PACS responses were stable over time, which is consistent with that of Simpson et al., who also investigated repeated, weekly PACS assessments among a population including Veterans and individuals with PTSD [31]. This is an important finding because it suggests that weekly monitoring of self-report craving does not escalate or change the craving outcome itself for a sample of Veterans with co-occurring conditions.

Our PACS scores among AUDIT-C $(+)$ Veterans were generally lower than in other published studies. Yoon et al., for example, used the PACS to examine alcohol craving among Veterans with lifetime alcohol dependence and reported PACS scores falling within the low to mod- erate craving range [44], but our average PACS score over $6 \mathrm{wk}$ for the AUDIT-C $(+)$ Veterans (including CC and MHD $\pm \mathrm{mTBI}$ ) was $8.7 \pm 0.6$ (where 30 is the maximum score), which falls within the moderate craving range. Simpson et al. also reported a higher average PACS score $(11.4 \pm 8.4)$ in a sample that included Veterans and other individuals with and without PTSD who were monitored weekly for self-reported alcohol craving [31]. Chakravorty et al. reported an average PACS score of $15.7 \pm 7.2$ for a population that included Veterans [12]. Our disparate findings could be due to the use of the AUDIT-C(+) as a screening tool in the current study, compared with the Simpson et al. and Chakravorty et al. studies, which included patients who met criteria for AUD. Therefore, Veterans in our study may have lower levels of alcohol addiction severity than those studied by Simpson et al. and Chakravorty et al. Furthermore, the PACS responses reported by Simpson et al. were obtained via the telephone through the use of automated IVR technology. The use of IVR technology may afford more perceived anonymity than in our study, in which research staff completed the PACS and AUDIT-C questionnaires with participants over the telephone.

\section{LIMITATIONS AND FUTURE DIRECTIONS}

Although preliminary, these findings are important because they are the first to characterize frequency and severity of alcohol cravings among Veterans with mTBI and co-occurring MHDs. There are several study limitations that should be considered when interpreting the results. The sample recruited for additional participation was a convenience sample and as such may underestimate the contribution of co-occurring conditions to the frequency and severity of alcohol cravings in Veterans. The total sample size of 48 and the even smaller size of the subgroups limit the generalizability of findings. In addition, to maintain sufficient subgroup size, the MHD \pm mTBI groups included individuals with a combination of depression symptoms, anxiety symptoms, PTSD, and mTBI. Consequently, we were unable to identify the individual effect of mTBI, depression, anxiety, or PTSD symptoms on alcohol craving. Also, there was a significant difference in employment status between the MHD and MHD $+\mathrm{mTBI}$ groups in that a greater proportion of individuals in the MHD group relative to the MHD + mTBI were working. 
In this study, a research assistant administered the PACS via the telephone. Although a similar approach has been used in the literature [31], we did not compare administration of the PACS through pencil and paper relative to telephone administration. Future studies are needed to further investigate the measurement properties of the PACS over the telephone because this method has potential utility for providers to capture programmatic outcomes.

Future research should include a sufficiently large sample of each subgroup so that the contribution of each symptom or disorder on alcohol craving can be examined. A larger study sample would enable us to distinguish between the effects of MHD and mTBI on alcohol craving and would also allow us to determine whether important covariates such as time since injury and sociodemographic factors influence the relationship. Future research should also examine how PACS administration methods (in-person pencil and paper vs telephone) may have influenced alcohol craving reporting. We were unable to collect information about predeployment mental health history including substance use disorders, but predeployment information would also be helpful in future research to determine whether incurring an mTBI mediated or led to postinjury alcohol use or alcohol craving behaviors.

Finally, because our preliminary data revealed lower PACS scores than in previous studies of alcohol craving among individuals with diagnosed AUD [12,31], in future studies we plan to expand our assessments of alcohol use and addiction/dependence. We also plan to include Veterans with more severe AUD, and to do so, we may recruit Veterans from VA addiction treatment centers. This approach is supported by a published finding that alcohol craving among Veterans is significantly associated with higher alcohol use and severity of alcohol dependence [44].

\section{CONCLUSIONS}

These descriptive findings are the first to characterize frequency and severity of alcohol craving among populations that include mTBI, which is of considerable interest given recent evidence indicating that $\mathrm{mTBI}$ increases risk for developing AUD among military personnel [7]. Therefore, the finding that Veterans with co-occurring MHDs with and without mild TBI have more severe and more frequent alcohol craving is important for develop- ing treatment plans for clinical rehabilitation. This study provides the necessary first step in laying the foundation for future research in the area of alcohol craving among populations with co-occurring MHDs and mTBI, which is essential to developing better treatment as well as treatment evaluation.

\section{ACKNOWLEDGMENTS}

\section{Author Contributions:}

Study concept and design: A. A. Herrold, N. Jordan, R. A. Chambers, T. L. Pape.

Acquisition of data: A. A. Herrold, S. Jenkins, X. Li.

Analysis and interpretation of data: A. A. Herrold, T. Mallinson, X. Li.

Preparation of manuscript: N. Jordan, W. M. High, T. Mallinson, B. Smith, C. T. Evans, X. Li, T. L. Pape, J. Babcock-Parziale. Drafting of manuscript: A. A. Herrold.

Financial Disclosures: The authors have declared that no competing interests exist.

Funding/Support: This material was based on work supported by the Edward Hines, Jr. VA Hospital (grants VAH CMC3 LIP 42-130 and VA HSR\&D SDR-08-377).

Institutional Review: This study was approved by the Edward Hines, Jr. VA Hospital Institutional Review Board.

Participant Follow-Up: The authors do not plan to inform participants of the publication of this study because permission to recontact the participants was not part of the Institutional Review Boardapproved research protocol.

Disclaimer: The views expressed in this article are those of the authors and do not necessarily reflect the position or policy of the VA or U.S. Government.

\section{REFERENCES}

1. Burnett-Zeigler I, Ilgen M, Valenstein M, Zivin K, Gorman L, Blow A, Duffy S, Chermack S. Prevalence and correlates of alcohol misuse among returning Afghanistan and Iraq veterans. Addict Behav. 2011;36(8):801-6. [PMID:21482030] http://dx.doi.org/10.1016/j.addbeh.2010.12.032

2. Seal KH, Cohen G, Waldrop A, Cohen BE, Maguen S, Ren L. Substance use disorders in Iraq and Afghanistan veterans in VA healthcare, 2001-2010: Implications for screening, diagnosis and treatment. Drug Alcohol Depend. 2011; 116(1-3):93-101. [PMID:21277712] http://dx.doi.org/10.1016/j.drugalcdep.2010.11.027

3. Calhoun PS, Elter JR, Jones ER Jr, Kudler H, StraitsTröster K. Hazardous alcohol use and receipt of risk-reduction counseling among U.S. veterans of the wars in Iraq and Afghanistan. J Clin Psychiatry. 2008;69(11):1686-93. 


\section{[PMID:19012816]}

http://dx.doi.org/10.4088/JCP.v69n1103

4. Carlson KF, Nelson D, Orazem RJ, Nugent S, Cifu DX, Sayer NA. Psychiatric diagnoses among Iraq and Afghanistan war veterans screened for deployment-related traumatic brain injury. J Trauma Stress. 2010;23(1):17-24. [PMID:20127725]

5. Graham DP, Cardon AL. An update on substance use and treatment following traumatic brain injury. Ann N Y Acad Sci. 2008;1141:148-62. [PMID:18991956] http://dx.doi.org/10.1196/annals.1441.029

6. Polusny MA, Kehle SM, Nelson NW, Erbes CR, Arbisi PA, Thuras P. Longitudinal effects of mild traumatic brain injury and posttraumatic stress disorder comorbidity on postdeployment outcomes in national guard soldiers deployed to Iraq. Arch Gen Psychiatry. 2011;68(1):79-89. [PMID:21199967] http://dx.doi.org/10.1001/archgenpsychiatry.2010.172

7. Miller SC, Baktash SH, Webb TS, Whitehead CR, Maynard C, Wells TS, Otte CN, Gore RK. Risk for addictionrelated disorders following mild traumatic brain injury in a large cohort of active-duty U.S. airmen. Am J Psychiatry. 2013;170(4):383-90. [PMID:23429886] http://dx.doi.org/10.1176/appi.ajp.2012.12010126

8. Seal KH, Metzler TJ, Gima KS, Bertenthal D, Maguen S, Marmar CR. Trends and risk factors for mental health diagnoses among Iraq and Afghanistan veterans using Department of Veterans Affairs health care, 2002-2008. Am J Public Health. 2009;99(9):1651-58. [PMID:19608954] http://dx.doi.org/10.2105/AJPH.2008.150284

9. Corrigan JD. Substance abuse as a mediating factor in outcome from traumatic brain injury. Arch Phys Med Rehabil. 1995;76(4):302-9. [PMID:7717829] http://dx.doi.org/10.1016/S0003-9993(95)80654-7

10. Jorge RE, Starkstein SE, Arndt S, Moser D, CrespoFacorro B, Robinson RG. Alcohol misuse and mood disorders following traumatic brain injury. Arch Gen Psychiatry. 2005;62(7):742-49. [PMID:15997015]

http://dx.doi.org/10.1001/archpsyc.62.7.742

11. Bottlender M, Soyka M. Impact of craving on alcohol relapse during, and 12 months following, outpatient treatment. Alcohol Alcohol. 2004;39(4):357-61. [PMID:15208171] http://dx.doi.org/10.1093/alcalc/agh073

12. Chakravorty S, Kuna ST, Zaharakis N, O'Brien CP, Kampman KM, Oslin D. Covariates of craving in actively drinking alcoholics. Am J Addict. 2010;19(5):450-57. [PMID:20716308] http://dx.doi.org/10.1111/j.1521-0391.2010.00067.x

13. Drapkin ML, Yusko D, Yasinski C, Oslin D, Hembree EA, Foa EB. Baseline functioning among individuals with posttraumatic stress disorder and alcohol dependence. J Subst Abuse Treat. 2011;41(2):186-92. [PMID:21546205] http://dx.doi.org/10.1016/j.jsat.2011.02.012
14. Driessen M, Schulte S, Luedecke C, Schaefer I, Sutmann F, Ohlmeier M, Kemper U, Koesters G, Chodzinski C, Schneider U, Broese T, Dette C, Havemann-Reinicke U; TRAUMAB-Study Group. Trauma and PTSD in patients with alcohol, drug, or dual dependence: A multi-center study. Alcohol Clin Exp Res. 2008;32(3):481-88. [PMID:18215214] http://dx.doi.org/10.1111/j.1530-0277.2007.00591.x

15. Kavanagh DJ, May J, Andrade J. Tests of the elaborated intrusion theory of craving and desire: Features of alcohol craving during treatment for an alcohol disorder. Br J Clin Psychol. 2009;48(3):241-54. [PMID:19364447] http://dx.doi.org/10.1348/014466508X387071

16. Schwab KA, Ivins B, Cramer G, Johnson W, Sluss-Tiller M, Kiley K, Lux W, Warden D. Screening for traumatic brain injury in troops returning from deployment in Afghanistan and Iraq: Initial investigation of the usefulness of a short screening tool for traumatic brain injury. J Head Trauma Rehabil. 2007;22(6):377-89. [PMID:18025970] http://dx.doi.org/10.1097/01.HTR.0000300233.98242.87

17. Tanielian T, Jaycox LH. Invisible wounds of war: Psychological and cognitive injuries, their consequences, and services to assist recovery. Santa Monica (CA): RAND Corporation; 2008.

18. Hoge CW, McGurk D, Thomas JL, Cox AL, Engel CC, Castro CA. Mild traumatic brain injury in U.S. Soldiers returning from Iraq. N Engl J Med. 2008;358(5):453-63. [PMID:18234750] http://dx.doi.org/10.1056/NEJMoa072972

19. Hibbard MR, Uysal S, Kepler K, Bogdany J, Silver J. Axis I psychopathology in individuals with traumatic brain injury. J Head Trauma Rehabil. 1998;13(4):24-39. [PMID:9651237] http://dx.doi.org/10.1097/00001199-199808000-00003

20. Sinha R, O’Malley SS. Craving for alcohol: Findings from the clinic and the laboratory. Alcohol Alcohol. 1999;34(2): 223-30. [PMID:10344782] http://dx.doi.org/10.1093/alcalc/34.2.223

21. Schneekloth TD, Biernacka JM, Hall-Flavin DK, Karpyak VM, Frye MA, Loukianova LL, Stevens SR, Drews MS, Geske JR, Mrazek DA. Alcohol craving as a predictor of relapse. Am J Addict. 2012;21(Suppl 1):S20-26. [PMID:23786506]

22. Keyes KM, Krueger RF, Grant BF, Hasin DS. Alcohol craving and the dimensionality of alcohol disorders. Psychol Med. 2011;41(3):629-40. [PMID:20459881] http://dx.doi.org/10.1017/S003329171000053X

23. Gordon SM, Sterling R, Siatkowski C, Raively K, Weinstein S, Hill PC. Inpatient desire to drink as a predictor of relapse to alcohol use following treatment. Am J Addict. 2006;15(3):242-45. [PMID:16923671] http://dx.doi.org/10.1080/10550490600626556 
24. Linacre JM. Sample size and item calibration (or person measure) stability. Rasch Meas Trans. 1994;7(4):328.

25. Chen WH, Lenderking W, Jin Y, Wyrwich KW, Gelhorn H, Revicki DA. Is Rasch model analysis applicable in small sample size pilot studies for assessing item characteristics? An example using PROMIS pain behavior item bank data. Qual Life Res. 2014;23(2):485-93. [PMID:23912855] http://dx.doi.org/10.1007/s11136-013-0487-5

26. Homaifar BY, Brenner LA, Gutierrez PM, Harwood JF, Thompson C, Filley CM, Kelly JP, Adler LE. Sensitivity and specificity of the Beck Depression Inventory-II in persons with traumatic brain injury. Arch Phys Med Rehabil. 2009;90(4):652-56. [PMID:19345782] http://dx.doi.org/10.1016/j.apmr.2008.10.028

27. Beck AT, Steer RA. Manual for the Revised Beck Depression Inventory. San Antonio (TX): Psychological Corp; 1987.

28. Beck AT, Steer RA. Manual for the Beck Anxiety Inventory. San Antonio (TX): Psychological Corp; 1988.

29. Beck AT, Epstein N, Brown G, Steer RA. An inventory for measuring clinical anxiety: Psychometric properties. J Consult Clin Psychol. 1988;56(6):893-97. [PMID:3204199] http://dx.doi.org/10.1037/0022-006X.56.6.893

30. Weathers FW, Ruscio AM, Keane TM. Psychometric properties of nine scoring rules for the Clinician-Administered Posttraumatic Stress Disorder Scale. Psychol Assess. 1999;11:124-33. http://dx.doi.org/10.1037/1040-3590.11.2.124

31. Simpson TL, Kivlahan DR, Bush KR, McFall ME. Telephone self-monitoring among alcohol use disorder patients in early recovery: A randomized study of feasibility and measurement reactivity. Drug Alcohol Depend. 2005; 79(2):241-50. [PMID:16002033]

http://dx.doi.org/10.1016/j.drugalcdep.2005.02.001

32. Flannery BA, Volpicelli JR, Pettinati HM. Psychometric properties of the Penn Alcohol Craving Scale. Alcohol Clin Exp Res. 1999;23(8):1289-95. [PMID:10470970] http://dx.doi.org/10.1111/j.1530-0277.1999.tb04349.x

33. Flannery BA, Poole SA, Gallop RJ, Volpicelli JR. Alcohol craving predicts drinking during treatment: An analysis of three assessment instruments. J Stud Alcohol. 2003;64(1): 120-26. [PMID:12608492]

34. Bush K, Kivlahan DR, McDonell MB, Fihn SD, Bradley KA. The AUDIT alcohol consumption questions (AUDITC): An effective brief screening test for problem drinking. Ambulatory Care Quality Improvement Project (ACQUIP). Alcohol Use Disorders Identification Test. Arch Intern Med. 1998;158(16):1789-95. [PMID:9738608]

http://dx.doi.org/10.1001/archinte.158.16.1789

35. Bradley KA, Bush KR, Epler AJ, Dobie DJ, Davis TM, Sporleder JL, Maynard C, Burman ML, Kivlahan DR. Two brief alcohol-screening tests From the Alcohol Use Disorders Identification Test (AUDIT): Validation in a female Veterans Affairs patient population. Arch Intern Med.
2003;163(7):821-29. [PMID:12695273]

http://dx.doi.org/10.1001/archinte.163.7.821

36. Reinert DF, Allen JP. The alcohol use disorders identification test: An update of research findings. Alcohol Clin Exp Res. 2007;31(2):185-99. [PMID:17250609] http://dx.doi.org/10.1111/j.1530-0277.2006.00295.x

37. Linacre JM. A user's guide to WINSTEPS ${ }^{\circledR}$ MINISTEP Rasch-model computer programs: Program manual 3.74.0 [Internet]; 2012. Available from: http://www.winsteps.com/ a/winsteps-manual-3740.pdf

38. Wright B, Masters G. Rating scale analysis. Chicago (IL): MESA Press; 1982.

39. Pesudovs K, Burr JM, Harley C, Elliott DB. The development, assessment, and selection of questionnaires. Optom Vis Sci. 2007;84(8):663-74. [PMID:17700331] http://dx.doi.org/10.1097/OPX.0b013e318141fe75

40. Wright BD. Reliability and separation. Rasch Meas Trans. 1996;9(4):472.

41. Wright BD, Linacre JM. Reasonable mean-square fit values. Rasch Meas Trans. 1994;8(370).

42. Pesudovs K, Gothwal VK, Wright T, Lamoureux EL. Remediating serious flaws in the National Eye Institute Visual Function Questionnaire. J Cataract Refract Surg. 2010;36(5):718-32. [PMID:20457362] http://dx.doi.org/10.1016/j.jcrs.2009.11.019

43. Hedecker D, Gibbons RD. Longitudinal data analysis. Hoboken (NJ): John Wiley \& Sons; 2006.

44. Yoon G, Kim SW, Thuras P, Grant JE, Westermeyer J. Alcohol craving in outpatients with alcohol dependence: Rate and clinical correlates. J Stud Alcohol. 2006; 67(5):770-77. [PMID:16847547]

Submitted for publication July 30, 2013. Accepted in revised form July 17, 2014.

This article and any supplementary material should be cited as follows:

Herrold AA, Jordan N, High WM, Babcock-Parziale J, Chambers RA, Smith B, Evans CT, Li X, Mallinson T, Jenkins S, Pape TL. Alcohol use and craving among Veterans with mental health disorders and mild traumatic brain injury. J Rehabil Res Dev. 2014;51(9):1397-1410. http://dx.doi.org/10.1682/JRRD.2013.07.0170

ResearcherID/ORCID: Judi Babcock-Parziale, PhD: E-5862-2012; Trudy Mallinson, PhD, OTR/L, FAOTA: B-8392-2009

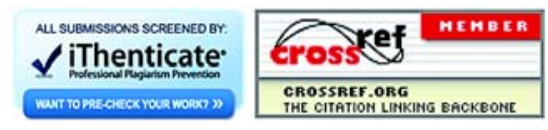


1410

JRRD, Volume 51, Number 9, 2014 
